\title{
Observation of Conical Waves in Focusing, Dispersive, and Dissipative Kerr Media
}

\author{
P. Polesana, ${ }^{1,2}$ A. Couairon, ${ }^{3}$ D. Faccio, ${ }^{2}$ A. Parola,${ }^{2}$ M. A. Porras,${ }^{4}$ A. Dubietis, ${ }^{1}$ A. Piskarskas, ${ }^{1}$ and P. Di Trapani ${ }^{1,2}$ \\ ${ }^{1}$ Department of Quantum Electronics, Vilnius University, Sauletekio Avenue 9, LT-10222 Vilnius, Lithuania \\ ${ }^{2}$ CNISM and Department of Physics and Mathematics, University of Insubria, Via Valleggio 11, 22100 Como, Italy \\ ${ }^{3}$ Centre de Physique Théorique, École Polytechnique, CNRS, F-91128, Palaiseau, France \\ ${ }^{4}$ Departamento de Fisica Aplicada, Universidad Politécnica de Madrid, Rios Rosas 21, E-28003 Madrid, Spain
}

(Received 18 May 2007; published 27 November 2007)

\begin{abstract}
Excitation of unbalanced-Bessel beams by a gradual increase of nonlinearity in a water sample outlines the achievement of the first ever observed quasimonochromatic wave packet that propagates stably for hundreds of Rayleigh lengths in a focusing and dispersive Kerr medium, i.e., in the absence of spectral broadening and conical emission. A modulational instability analysis reveals the key role of nonlinear dissipation in quenching the growth of spatiotemporal unstable modes.
\end{abstract}

PACS numbers: 42.65.Jx, 42.65.Sf

Since the earliest works on self-focusing, the hunt for intense optical beams that propagate stationarily in the presence of a fast, focusing, nonlinear response has signaled numerous milestones in nonlinear optics, the research being of major importance for all-optical signal processing, frequency conversion, remote ranging, control of electrical discharge, etc. Spatial solitons are often considered as the ideal tool for these applications [1]. In bulk Kerr media, however, the mechanism that stabilizes solitons against collapse causes also relevant nonlinear losses (NLL), which quench stationarity owing to limited solitonpower content [2]. Moreover, spatial solitons as any bellshaped, spatially localized, quasimonochromatic nonlinear solution suffer from modulational instability growing from the nonlocalized temporal (i.e., longitudinal) dimension, as shown by numerical simulations for the case of nanosecond filaments in gases [3] and by experiments for the $\chi^{(2)}$ spatial-solitons regime [4]. The NLL problem can be circumvented if nonlinear unbalanced-Bessel beams (NLUBB) are considered instead of solitons [5]. These are peculiar, propagation-invariant, conical wave packets, whose intense, narrow, interference hot core dissipates energy via NLL being simultaneously refilled by the incoming power from an extended energy reservoir, which is spontaneously formed during ultrashort laser pulse filamentation in Kerr media [6,7]. Bessel-like beams, however, have been demonstrated to suffer from important spatiotemporal instability, which prevents the achievement of the desired truly stationary regime [8].

In this Letter we report on the first ever observed long range stability of a subdimensional (e.g., quasimonochromatic) nonlinear wave propagating in a bulk, threedimensional material. The result is obtained by quenching the instability of quasimonochromatic Bessel-like waves via suitable control of the growth of their intensity inside the sample, so that a smooth transition between the linear and the nonlinear regime is obtained. In this case the beam dynamics is attracted by the monochromatic NLUBB solution, which here we show to be robust with respect to spatiotemporal instability.
In the experiment we used $10 \mu \mathrm{J}, 200 \mathrm{fs}$, and $527 \mathrm{~nm}$ laser pulses delivered by a Nd:glass laser system (TWINKLE, Light Conversion Ltd.). Pulsed Bessel beams (BB) were generated by launching a Gaussian beam of radius $0.8 \mathrm{~mm}$ at $1 / e^{2}$ into a $\mathrm{BK} 7$ glass axicon $\left(n_{\mathrm{BK} 7}=\right.$ 1.52) of base angle $2^{\circ} 50^{\prime}$. The resulting profile leads to a central interference spike with diameter of $15 \mu \mathrm{m}$ (between the zeros of Bessel function), which grows due to linear propagation in air reaching its maximum intensity at a distance $z=20 \mathrm{~mm}$ from the axicon. In a first run of measurements, we used a $30 \mathrm{~mm}$ long cuvette filled with water $\left(n_{\text {water }}=1.33\right)$, the entrance window of which was located at $z_{m}=20 \mathrm{~mm}$ from the axicon. With this setting, the wave packet undergoes a sharp transition between the linear and nonlinear regimes. Pulse lengthening is negligible due to the fact that the dispersion length $(36 \mathrm{~cm})$ is much longer than the sample length. Therefore the pulse may be considered as quasimonochromatic. In a second experiment, we used a $50 \mathrm{~mm}$ long cuvette with entrance window in contact with the axicon and output window at the same position as in the first experiment so as to force a smooth transition to the nonlinear propagation regime in the first $20 \mathrm{~mm}$ of the cuvette. Hereafter we will refer to abrupt or soft input conditions for these two cases. Because of their small thickness $(1 \mathrm{~mm})$, the cuvette walls have no impact on the nonlinear propagation and weak impact on the pulse dispersion [9]. Beyond the cuvette we measured the angularly resolved spectra of the propagated field by placing an imaging spectrometer in the Fourier plane of a $50 \mathrm{~mm}$ achromatic lens. The obtained $\left(k_{\perp}, \lambda\right)$ spectra were digitalized by an ANDOR iDus camera.

An insight of the phenomena occurring inside the cuvette was obtained through numerical simulations. Our code solves the nonlinear equation for the envelope $\mathcal{E}(r, t, z)$ of the laser field with central frequency $\omega_{0}$ and accounts for diffraction, dispersion, self-focusing, and nonlinear losses [10]:

$$
2 k_{0} \frac{\partial \mathcal{E}}{\partial z}=i \nabla_{\perp}^{2} \mathcal{E}-i k_{0} k^{\prime \prime} \frac{\partial^{2} \mathcal{E}}{\partial t^{2}}+2 k_{0} N(\mathcal{E})
$$




$$
N(\mathcal{E})=i \frac{\omega_{0}}{c} n_{2}|\mathcal{E}|^{2} \mathcal{E}-\frac{\beta_{K}}{2}|\mathcal{E}|^{2 K-2} \mathcal{E},
$$

where $\nabla_{\perp}^{2} \equiv \partial_{r}^{2}+(1 / r) \partial_{r}, r$ is the radial coordinate, and $t$ refers to the local time. From Eq. (12) in Ref. [11], we obtained the dispersion $k(\omega)$, the wave number $k_{0} \equiv$ $k\left(\omega_{0}\right)$, and the coefficient $k^{\prime \prime}=\left.\partial_{\omega}^{2} k(\omega)\right|_{\omega_{0}}=560 \mathrm{fs}^{2} / \mathrm{cm}$. The nonlinear refractive index of water $n_{2}=2.7 \times$ $10^{-16} \mathrm{~cm}^{2} /$ W was taken from Ref. [12]. Parameters $K=$ 4 and $\beta_{K}=2.4 \times 10^{-36} \mathrm{~cm}^{5} / \mathrm{W}^{3}$ for nonlinear losses were determined in Ref. [13]. The depletion of the medium calculated by means of the extended model Eqs. (39)-(44) in Ref. [14] does not exceed $5 \times 10^{18} \mathrm{~cm}^{-3}$ justifying its neglect. The wavelength, duration, energy, and radius at beam waist of the Gaussian input pulse are the same as in the experiment. We model the effect of the axicon by a radial phase modulation $\phi(r)=k_{0} r \sin (\theta)$ of the Gaussian input beam.

Figure 1 shows the measured $\left(k_{\perp}, \lambda\right)$ spectra [Figs. 1(a)$1(\mathrm{c})$ ], as well as their counterparts obtained by simulation [Figs. 1(d)-1(f)]. Figures 1(a) and 1(d) were obtained by lowering the energy to keep the propagation linear and show the spectrum of the input BB featured by a transverse Bessel wave vector $k_{B}=k_{0} \sin \theta$, where $\theta \simeq \gamma\left(n_{\mathrm{BK} 7}-\right.$ $1) / n_{\text {water }}=19 \mathrm{mrad}$ is the angle imposed by the axicon that we used. Note the slight broadening in the tranverse plane with respect to the ideal case, due to finite beam apodization. Figures 1(b) and 1(e) display the far-field spectrum of the pulse that experienced an abrupt linearnonlinear transition; the spectra exhibit an axial radiation extending from 500 to $560 \mathrm{~nm}$ and nearly hyperbolic branches featuring a broad conical emission with a wave
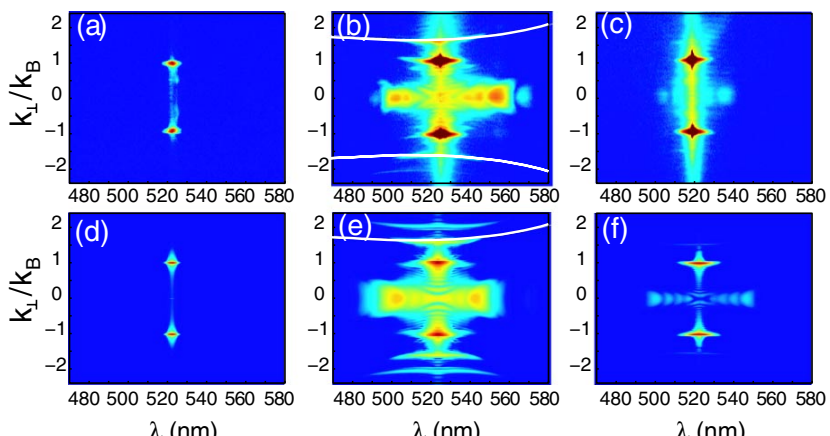

$\lambda(\mathrm{nm})$

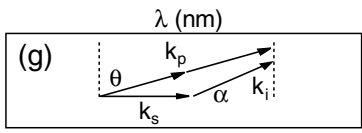

FIG. 1 (color online). Angularly resolved $\operatorname{spectra}\left(k_{\perp}, \lambda\right)$ for different propagation configurations; 7 decades are plotted in log scale. Experimental (a) and simulated (d) linear propagation of the input BB. Experimental (b) and simulated (e) nonlinear propagation in water with an abrupt transition to the water cell; the white curves fitting the conical emission correspond to the longitudinal phase matching condition shown in $(\mathrm{g})$. Experimental (c) and simulated (f) nonlinear propagation in water in the case of soft input conditions. number gap of $\sim 1.5 k_{B}$. Figures 1 (c) and 1(f) show the spectrum in the case of the smooth nonlinear propagation in the longest cuvette in contact with the axicon. Only minor changes with respect to Figs. 1(a) and 1(d) are visible: there are no conical branches and a very weak axial emission. The spectral features obtained by simulation are in excellent agreement with the measurements.

To interpret these features, note that for the abrupt linear-nonlinear transition a good fit of the hyperbolic branches is given by $k_{\perp}=k_{i} \sin \alpha$, where the angle $\alpha(\omega)$ satisfies the longitudinal phase matching condition depicted in Fig. 1(g) between two pump photons $\mathbf{k}_{p}$ from the Bessel cone of angle $\theta$, a signal $\mathbf{k}_{s}\left(\omega_{0}+\Delta \omega\right)$ from axial radiation, and the conical idler $\mathbf{k}_{i}\left(\omega_{0}-\Delta \omega\right)$ : $k_{i} \cos \alpha=2 k_{p} \cos \theta-k_{s}$ [8]. The result indicates therefore that the abrupt transition leads to important four-wave spatiotemporal dynamics, which takes place while fulfilling longitudinally phase matching constraint. Surprisingly, for the smooth linear-nonlinear transition a longer nonlinear propagation does not enhance the features observed in the case of the abrupt input condition but leads to the quenching of both axial radiation and hyperbolic branches. This indicates a strong difference in the nature of the nonlinear regime that is controlled by the abruptness of nonlinearity. The quenching is interpreted as due to a spontaneous transformation of the input $\mathrm{BB}$ into a stationary and nonlinear stable field distribution, characterized by the absence of temporal dynamics.

Further numerical simulations allow us to describe the field transformation inside the cuvette, which cannot be accessed by experiments. Figure 2(a) shows a comparison between the peak fluence in the case of abrupt (dashed line) and soft (solid) input in the Kerr medium. The abrupt input condition gives rise to strong oscillations, attributed to the beating between the $\mathrm{BB}$ and the axial radiation [15]. In contrast the solid curve shows that the fluence evolution in the case of a soft input remains smooth until the end of the cuvette, in agreement with the absence of any significant axial radiation. Figure 2(b) shows the temporal pulse shape at $r=0$ as a function of the propagation distance in the case of an abrupt transition: the pulse undergoes dramatic reshaping which leads to multiple splittings as indicated by the sequence of $V$-shaped patterns. The two arms of each $V$ pattern correspond to a couple of split pulses traveling at group velocities that are subluminal and superluminal with respect to the input luminal group velocity $1 / k^{\prime}\left(\omega_{0}\right)$, as indicated by the slopes of the $V$-shaped arms. In the case of a soft input [Fig. 2(c)], the temporal envelope remains regular and Gaussian-like during the nonlinear propagation through the whole cell, highlighting subdimensional dynamics where temporal transformation is absent and radial reshaping prevails.

The numerical simulations are also analyzed by plotting the trajectories representing the wave evolution in the phase space shown in Fig. 3, defined by the squared half 

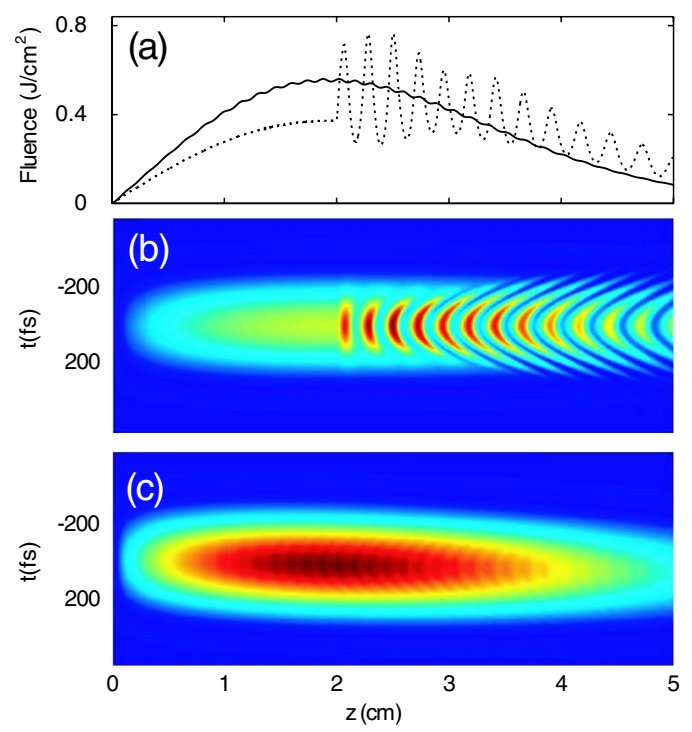

FIG. 2 (color online). (a) Peak fluence versus propagation distance obtained from simulation. Dashed line: abrupt linearnonlinear transition at $z_{m}=20 \mathrm{~mm}$. Solid line: soft transition to the nonlinear regime. (b),(c) On axis intensity distribution showing the temporal dynamics in the $(z, t)$ plane for an abrupt (b) and a soft (c) input condition.

width at half maximum (HWHM) of the beam and peak intensity. In order to look for a possible attractor of the dynamics, we plotted in this plane the black dashed curve representing the family of monochromatic stationary solutions to Eqs. (1) and (2) which have the same cone angle as that set by the axicon in the experiment. These solutions are of the form $\mathcal{E}=a(r) \exp (-i \delta z)$, where $\delta \simeq k_{0} \theta^{2} / 2$ denotes the axial wave vector shift with respect to $k_{0}$, have a typical amplitude profile $|a(r)|$ as in the inset of Fig. 3 and are known as the NLUBB [5].

The dotted curve refers to the abrupt input condition: after the linear propagation which keeps constant the beam



FIG. 3 (color online). Phase space trajectories of the numerically simulated beams obtained by collecting the peak intensities and HWHM beam width along propagation. Dotted curve: abrupt input; solid curve: soft input. The bold dashed curve is the theoretically predicted set of attractors (see the text). The inset shows the typical profile (normalized) of these NLUBB attractors. radius, the nonlinear propagation follows an oscillating trajectory spanning a wide area of the phase space. In contrast the trajectory for the soft input condition (solid curve) is confined in a reduced volume and represents a slow motion along the family of NLUBBs featured by the cone angle fixed in the experiment. The overlap of the solid and the bold dashed curves shows that these NLUBBs represent attractors of the dynamics of the Bessel beam in dispersive Kerr media with nonlinear losses.

The quenching of the axial and conical emissions obtained for soft input conditions, which forces the beam parameters to remain close to those of a NLUBB at each propagation distance, indicates these solutions as being potentially robust against spatiotemporal modulational instability, a finding that advocates for a verification.

With the dimensionless quantities $\rho=\sqrt{2 k_{0}} \delta r, \xi=\delta z$, $\tau=\sqrt{2 \delta / k^{\prime \prime}} t$, and $\mathcal{A}=\mathcal{E} / \mathcal{E}_{0}$, we rewrite Eq. (1) as

$$
\partial_{\xi} \mathcal{A}=i \Delta_{\rho} \mathcal{A}-i \partial_{\tau}^{2} \mathcal{A}+i g|\mathcal{A}|^{2} \mathcal{A}-\gamma|\mathcal{A}|^{2 K-2},
$$

where $\Delta_{\rho}=\partial_{\rho}^{2}+(1 / \rho) \partial_{\rho}, g=\omega_{0} n_{2} \mathcal{E}_{0}^{2} / c \delta$, and $\gamma=$ $\beta_{K} \mathcal{E}_{0}^{2 K-2} / 2 \delta$. For the intensity range $0.3-3 \mathrm{TW} / \mathrm{cm}^{2}$ displayed in Fig. 3, these parameters vary in the range $0.34<$ $g<3.4$ and $1.1 \times 10^{-3}<\gamma<1$. 1 . NLUBBs consist in a three parameter family $(K, g$, and $\gamma)$ of monochromatic stationary solutions to Eq. (3) in the form $\mathcal{A}=a_{0}(\rho) e^{-i \xi}$, satisfying $a_{0}(0)=1$ and $a_{0}(\infty)=0$ [5]. Following a standard procedure [16], we introduce perturbed NLUBBs

$$
\mathcal{A}=\left[a_{0}(\rho)+u(\rho) e^{-i \Omega \tau-i \kappa \xi}+v^{*}(\rho) e^{i \Omega \tau+i \kappa^{*} \xi}\right] e^{-i \xi}
$$
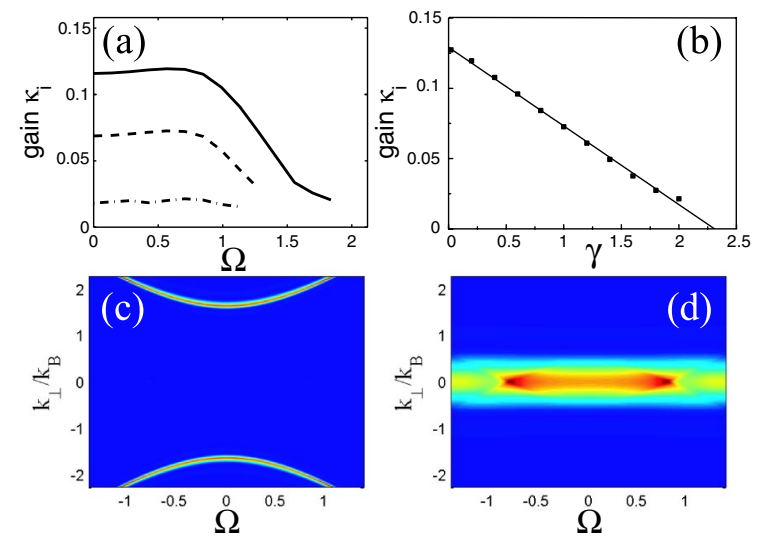

FIG. 4 (color online). Features of the modulational instability of NLUBB computed for $K=4$ and $g=2$. (a) Gain of the pair of unstable modes for the perturbed NLUBB vs perturbation frequency for $\gamma=0.02$ (solid curve), 0.1 (dashed curve), and 0.2 (dash-dotted curve). (b) Maximum gain over the perturbation frequency as a function of $\gamma$. (c),(d) $\left(k_{\perp}, \Omega\right)$ spectrum of the $u$ and $v$ perturbations of the NLUBB for $\gamma=0.01$. The $u$ and $v$ components correspond to conical (c) and axial (d) radiations, respectively. 
into Eq. (3) to obtain, after linearization, the eigenvalue problem

$$
\begin{gathered}
\kappa u=-\left(H+i \Gamma_{K}\right) u-a_{0}^{2}\left(g+i \Gamma_{K-1}\right) v, \\
\kappa v=a_{0}^{* 2}\left(g-i \Gamma_{K-1}\right) u+\left(H-i \Gamma_{K}\right) v,
\end{gathered}
$$

where $H=\Delta_{\rho}+\Omega^{2}+2 g\left|a_{0}\right|^{2}+1, \quad \Gamma_{J}=J \gamma\left|a_{0}\right|^{2 J-2}$ with $J=K, K-1$ and boundary conditions $u(\rho) \rightarrow 0$, $v(\rho) \rightarrow 0$ as $\rho \rightarrow \infty$. The instability of the NLUBB is determined by the existence of modes growing exponentially as $\exp \left(\kappa_{i} \xi\right)$ when the eigenvalue $\kappa$ has positive imaginary part $\kappa_{i}>0$ (i.e., gain). Equations (5) and (6) were solved numerically for each perturbation frequency $\Omega=\left(\omega-\omega_{0}\right) \sqrt{k^{\prime \prime} / 2 \delta}$ to determine the gain $\kappa_{i}(\Omega)$ and the spectral features of the instability modes.

Figure 4(a) shows the gain computed for the NLUBB with $K=4, g=2$, and different nonlinear losses coefficients $\gamma$. For each frequency a single couple of modes is found to be unstable: $(\kappa, u, v)$ and its symmetric $\left(-\kappa^{*}, v^{*}\right.$, $\left.u^{*}\right)$. By increasing the nonlinear losses, the gain becomes smaller, as shown by the maxima of the gain curves collected in Fig. 4(b). Unstable modes with small gain exhibit a slow exponential decay as $\exp (-\beta \rho)$ where $\beta \sim \kappa_{i} / 2$. Therefore, in the numerical calculation in a finite radial box of radius $\rho=L$, the minimum growth rate which may be reliably calculated scales as $\kappa_{i, \min }=2 / L$. The smallest gain on Fig. 4(b) corresponds to this limit with a box size of $L=400$ at the maximum accessible resolution on our computers (4000 points). A fit of the computed data, however, lets us foresee that the gain vanishes at a given NLL value. This indicates that the effect of nonlinear losses is to stabilize NLUBBs. This result extends the finding of Ref. [5] to the case of spatiotemporal perturbations. The $\left(k_{\perp}, \Omega\right)$ spectrum of the growing modes is obtained by performing a Hankel transform of the unstable modes $[u(\rho), v(\rho)]$ computed for each $\Omega$. Generic features of the $\left(k_{\perp}, \Omega\right)$ spectra are shown in Figs. 4(c) and 4(d) for the pair of unstable modes obtained for $\gamma=0.01$. Figure 4(c) shows the angularly resolved spectrum of the $u$ component in the unstable mode, consisting of a conical emission with gap $\left(1.5 k_{B}\right)$ in transverse wave vector, while Fig. 4(d) shows the $\left(k_{\perp}, \Omega\right)$ spectrum of the $v$ component in the perturbation, characterized by an axial emission. Note the good agreement between the angular spectrum of the unstable NLUBB mode and that of the growing perturbation in Figs. 1(b) and 1(e). The modulational instability analysis shows that NLUBBs become stable with respect to spatiotemporal perturbations for sufficiently large material NLL coefficients and large peak intensity.

In conclusion, the quenching of the axial and conical emissions obtained for soft input conditions is due to a slow transient dynamics of the beam in the sole transverse coordinate, without spatiotemporal modulational instabil- ity. We interpret the nonlinear propagation as an evolution along a family of exact subdimensional stationary solutions in the form of nonlinear dissipative Bessel beams (NLUBBs) supported by the focusing dispersive medium. Stabilization is achieved in the presence of large nonlinear losses and appropriate excitation of the NLUBB. This result opens up new perspectives for applications which require long range light matter interactions, such as micromachining or waveguide writing. Furthermore, the results, based on the analysis of the quite universal nonlinear Schrödinger equation, apply also in other fields as, e.g., nonlinear hydrodynamics, plasma physics, or BoseEinstein condensates [17].

P.P. acknowledges the support from Sixth EU Framework Programme Contract No. MEST-CF-2004008048 (ATLAS). P. D. T. acknowledges the support from Marie Curie Chair project STELLA, Contract No. MEXCCT-2005-025710. D. F. and P. D. T. acknowledge financial support from CNISM, Innesco project.

[1] G. I. Stegeman, D. N. Christodoulides, and M. Segev, IEEE J. Sel. Top. Quantum Electron. 6, 1419 (2000).

[2] B. A. Malomed, D. Mihalache, F. Wise, and L. Torner, J. Opt. B 7, R53 (2005).

[3] T. A. Niday, E. M. Wright, M. Kolesik, and J. V. Moloney, Phys. Rev. E 72, 016618 (2005).

[4] S. D. Jenkins, D. Salerno, S. Minardi, G. Tamošauskas, T. A. B. Kennedy, and P. Di Trapani, Phys. Rev. Lett. 95, 203902 (2005).

[5] M. A. Porras, A. Parola, D. Faccio, A. Dubietis, and P. Di Trapani, Phys. Rev. Lett. 93, 153902 (2004).

[6] A. Dubietis, E. Gaižauskas, G. Tamošauskas, and P. Di Trapani, Phys. Rev. Lett. 92, 253903 (2004).

[7] D. Faccio, M. A. Porras, A. Dubietis, F. Bragheri, A. Couairon, and P. Di Trapani, Phys. Rev. Lett. 96, 193901 (2006).

[8] A. Dubietis, P. Polesana, G. Valiulis, A. Stabinis, P. Di Trapani, and A. Piskarskas, Opt. Express 15, 4168 (2007).

[9] F. Bragheri et al., Phys. Rev. A 76, 025801 (2007).

[10] A. Couairon, E. Gaižauskas, D. Faccio, A. Dubietis, and P. Di Trapani, Phys. Rev. E 73, 016608 (2006).

[11] A. G. Van Engen, S. A. Diddams, and T. S. Clement, Appl. Opt. 37, 5679 (1998).

[12] K. J. Witte, M. Galanti, and R. Volk, Opt. Commun. 34, 278 (1980).

[13] A. Dubietis, A. Couairon, E. Kučinskas, G. Tamošauskas, E. Gaižauskas, D. Faccio, and P. Di Trapani, Appl. Phys. B 84, 439 (2006).

[14] A. Couairon and A. Mysyrowicz, Phys. Rep. 441, 47 (2007).

[15] E. Gaižauskas, E. Vanagas, V. Jarutis, S. Juodkazis, V. Mizeikis, and H. Misawa, Opt. Lett. 31, 80 (2006).

[16] M. A. Porras, A. Parola, D. Faccio, A. Couairon, and P. Di Trapani, Phys. Rev. A 76, 011803(R) (2007).

[17] A. Alexandrescu and V. M. Perez-Garcia, Phys. Rev. A 73, 053610 (2006). 